\title{
New Thinking of Human Resource Management in the Age of Artificial Intelligence
}

\author{
Xinzi Li \\ Xi'an Peihua University, Xi’an, Shaanxi, 710125
}

Keywords: artificial intelligence; Human resources; Human resource management; new thinking

\begin{abstract}
The integration of artificial intelligence and existing technologies is increasing day by day, which solves a lot of problems for enterprises, which perform automation work behind the scenes by analyzing data sets. These ideas can reveal new ways to increase hiring success and deepen understanding of the company's employees from a strategic perspective. Combined with expertise in human resource management, this enhanced intelligence will help companies save costs, improve talent quality and improve the effectiveness of employee team plans. This paper takes the influence of artificial intelligence on human resource management as the core issue, and puts forward a new thinking of human resource management in the era of artificial intelligence.
\end{abstract}

\section{Introduction}

With the advent of the artificial intelligence era, enterprises are facing the opportunities and challenges brought by the global industrial restructuring. It can be said that a new round of scientific and technological revolution and industrial reform is emerging in the world. The rapid development of information technology and its deep integration with various fields of technology have triggered profound changes in economic and social development. Accompanied by a new generation of the breakthroughs and diffusion of information technology, flexible manufacturing, intelligent manufacturing, service, industrial Internet, 3D printing, mass customization, the whole life cycle management, etc., are the traditional development concept, development mode, development model produced disruptive, the influence of the revolutionary, and will reshape the global enterprise development pattern. Human work is gradually replaced by machines. In the future, smart factories require people to program and maintain networked machines and interpret complex data, forming teams with managers to work together. With the popularization of industrial robots, the boundary between workers and managers will become increasingly blurred in more and more enterprises. Because in a smart factory, everyone will be involved in analyzing and solving problems. For the current era of artificial intelligence, human resource management of Chinese enterprises must be re-interpreted and re-constructed. This paper will expound the idea and mode of strategic human resource management in the era of artificial intelligence.

\section{Artificial intelligence concept and its key subset}

Artificial Intelligence is short for AI. Artificial intelligence is the simulation of the information process of human consciousness and thinking. Artificial intelligence is not human intelligence, but it can think like a human and possibly surpass it. Artificial intelligence is a branch of computer science that seeks to understand the essence of intelligence and produce a new kind of intelligent machine that can respond in a similar way to human intelligence, with research in the field including robotics, language recognition, image recognition, natural language processing and expert systems.

Key subsets of AI include: (1) virtual assistant: technology that uses text and/or voice natural language processing to communicate with users and help them complete tasks (such as Siri); (2) augmented reality: create an enhanced version of interactive reality, using technology to overlay digital information into the existing environment; (3) machine learning: a technology that uses large amounts of data and algorithms to analyze and understand information, and then gives it the ability 
to learn; (4) cognitive computing: combine machine learning, data mining, pattern recognition and natural language processing, speech, vision and human-computer interaction to simulate the functions of human brain and help improve decision-making ability; (5) prediction analysis: use data, statistical algorithm and machine learning technology to predict future results.

\section{The impact of artificial intelligence on human resource management}

The development and improvement of artificial intelligence in the field of science and technology has profoundly changed the mode of production and life in modern society and also deeply affected human resource management. Artificial intelligence has challenged the traditional human resource management mode and promoted the development and reform of human resource management mode. The application of artificial intelligence has brought great advantages but also considerable challenges, and improper application will be counterproductive.

\subsection{Artificial intelligence reshaping the workplace}

As artificial intelligence takes over communications, office, collaboration and other functions, companies will pack lightly. In the era of sharing economy; people can work at any time and any place. How to bring together full-time employees to exert more potential, workplace redesign has become the focus of many enterprises. Work will be redefined and part of the task will be left to the robot. At the same time, a wide range of new tasks will be created, and employees will be motivated to work in unprecedented fields.

\subsection{Artificial intelligence replaces the repetitive work of personnel}

Artificial intelligence uses large amounts of past and present data to judge employees, so it can play a big role in a less volatile corporate environment. But when an enterprise wants to carry out technical innovation, or because of the emergency to launch new personnel strategy, still must rely on the human strength. There are a lot of human resource work. In the future, most "personnel" work and "six modules" work will be replaced by artificial intelligence methods and machine methods.

\subsection{Artificial intelligence human resource management will be more efficient}

Innovation has led to the development of intelligent robots that can do the simple things that humans can do. If used correctly, AI will enable companies to understand large amounts of structured and unstructured data and make faster, smarter decisions that drive cost savings and improve end-user experience. It's tempting to use AI to help recruit, manage and optimize employees. Using machine learning predictive analysis to improve efficiency, reduce operating costs, increase revenue, and improve talent quality offers possibilities.

\subsection{Artificial intelligence can optimize decisions of Human resource management}

Artificial intelligence technology also improves the management ability of enterprises, stimulates employees' enthusiasm and creativity, and enables organizations and employees to form a win-win relationship. Through the combination of artificial intelligence, employers can combine the past data analysis and behavior performance of candidates, and pay more attention to internal evaluation so that they can better match the position and the recruitment is more successful. The use of artificial intelligence in the training can effectively analyze staff's shortages and advantages, improve the effect of training for daily transaction performance appraisal; artificial intelligence can reduce human input, make the evaluation more accurate, but it is only part of the type of work, the innovative work, can not simply rely on daily affairs for performance management work still need to balance, to determine its value.

To sum up, although the specific functions may vary slightly depending on the industry and use situation, the management principles of AI for employees are similar, which will enable companies to make better hiring decisions, enable companies to manage their teams more effectively, and improve user's experience. 


\section{Innovative thinking of human resource management in the age of artificial intelligence}

Taking full advantage of artificial intelligence, HR departments are required to learn from and learn excellent benchmark enterprises in terms of organizational structure or building a new artificial intelligence team, and the ability of the whole staff team can adapt to the latest demands of the new era.

\subsection{To redefine the mission of human resource management}

The HR department needs to define itself as a team that assists management and employees in quickly transforming and adapting to a new mindset. HR should focus on business development and be a good business partner to promote the company's human resource management and efficiency through informatization. The HR team of Chinese enterprises has gone from closed way to open way, from functional orientation to business orientation, from satisfying internal communication to strengthening all-staff interconnection and strengthening external collaboration, and made innovative breakthroughs and improvements in product architecture, application functions, operation performance and user experience.

\subsection{To upgrade the core technology of human resource information management}

It needs to replace traditional systems with integrated cloud platforms to build a good digital infrastructure. Adhering to the 4S product concept of "supporting strategic decision-making, serving all-staff interconnection, enhancing social collaboration, and meeting timely supply", the vision of human resource management software is promoted from meeting the general needs of HR professionals in daily affairs to the height of enterprise strategy, culture and reform. It can develop a long-term human resource technology strategy that includes cloud enterprise resource planning platforms, applications, data analysis and a range of AI, case management and other solution tools to build a human resource digitalization team.

\subsection{Build the HR department into an expert network with strong business partners}

Human resources management localization is not being a regulator, controller, or assessed, our real value lies in to the passion of employees to pursue personal value realization and enterprise pursue innovation breakthrough vision combined effectively, activate a individual, organization, realize the value of the release, drive every employee achievement true self, in the process of achieving an organization's high performance achieve the high value transformation of HR to reconsider human resource organization mode, focus on staff experience, culture, analysis, and a new learning method, to make innovation a core strategy for human resources, to turn young employees to HR department.

\subsection{To establish a Wikipedia-style organizational structure}

There are five elements of a Wikipedia-style organization: (1) Embracing the speed of change: we need to think carefully about the ways in which slower-moving traditional business models are speeding up in the digital wave. (2) Taking talent mobility as the core value: many enterprises are doing this at present, such as rotating posts, dissolving and regrouping after the team is completed. Many projects also operate in this way facing the Internet. But when the stability of our team is amazing, we need to consider whether our team will be eliminated or replaced in the future. (3) Establishing accountability: empowering employees to make decisions and relying on the network for communication does not mean that employees are no longer responsible for the results. It is more and more difficult to monitor the process. Process feedback is more of a barometer. (4) Establishing an organizational performance team: arrange team visits, analyze and study the operation mode of the team, project and system with outstanding performance. (5) Adopting a continuous, feedback based performance management model: regular feedback enables employees to constantly re-set goals, improve projects, and feel valued for what they have done rather than for what they have been assigned.

In addition to the above, it is necessary to visit other enterprises to communicate and learn with 
excellent enterprises to understand the business trend of human resource management in other enterprises. The leading practice of enterprises stems from the innovative ideas formed by enterprise culture and business demands, rather than the pure theory in books.

\section{Conclusion}

All in all, human resources play an important role in enterprise responding to challenges in the era of artificial intelligence, which can help leaders and organizations adapt to technologies, help employees adapt to new work patterns, promote enterprises to adapt to technological changes, and promote the development and change of national policies and regulations. In the future, human resource management needs to apply more AI technologies, realize the interface of human resource model deep learning (position deep learning), look for professional talents on the network, gain insight into the needs of competitors, and evaluate models based on job experience and skill perception. Human resource managers must stand on the height of future development, adapt to the changes of various environmental situations, actively respond to the challenges of artificial intelligence, adapt to the development of informatization, and master the cutting-edge dynamics of artificial intelligence and other related technologies, so as to further clarify the real value of human resource management.

\section{References}

[1] Yanjun Long. Application of Artificial Intelligence (AI) Technology in Human Resource Management Information System [J]. Automation and Instrumentation, 2016(8):187-188.

[2] Liang Wang. On Improving Human Resource Management with Artificial Intelligence Technology [J]. Sinopec China, 2017(7):53-54.

[3] Yinlong Li, Xiaohong Wu. A Brief Analysis Impact of Artificial Intelligence on Human Resources Resource Management [J].Human Resource Management, 2018(3):400-401.

[4] Xiao Manhua. On the Value of Human Resource Practitioners in the Era of Artificial Intelligence [J]. Tax Payment, 2017(12):58-59.

[5] Qiqi Gao, Zhang Jiebin. On the Social Compensation and Individual Adaptation: Two Solutions to Unemployment in the Era of Artificial Intelligence [J]. Jiangxi Social Science, 2017(10):25-34.

[6] Zhe He. Thematic Research -Artificial Intelligence: Opportunities and Challenges: Social Transformation and Administrative Ethics in the Era of Artificial Intelligence: Can Machines Manage People? [J]. E-government, 2017(11):1-10. 